\title{
Review of: "Detrimental effect of diabetes and hypertension on the severity and mortality of COVID-19 infection: A multi-center case-control study from India"
}

Dafeng Liu

Potential competing interests: The author(s) declared that no potential competing interests exist.

This is a multi-center case-control study in India which examined effect of diabetes and hypertension on the severity and mortality of COVID-19 infection. The principal finding was that age, neutrophil\%, and diabetes status were significantly associated with severe COVID-19 infection. After adjusting for age, patients with diabetes were 2.46 times more likely to have severe disease and 2.11 times more likely to have a fatal outcome.

However, no evidence for hypertension modifying the COVID-19 outcomes in diabetic patients was found. Significant limitation in the study was that in the retrospective record-based study a significant amount of data due to digitization was lost. Going forward, an effort should be made to achieve optimal glycemic control in diabetic patients to prevent increased severity and mortality due to COVID-19. Telemedicine is also a promising alternative to conventional hospital appointments for diabetic patients to access healthcare facilities especially in these trying times.

There are several concerns with this study which include the following:

1. Since this was a retrospective record-based study, a significant amount of data due to digitization was lost, such as glycemic control level, duration of diabetes, acute and chronic complications of diabetes, hypoglycemic drugs and so on. In addition to considering the diabetes itself affecting the severity and prognosis of COVID-19, it is also necessary to consider above diabetes related factors affecting the severity and prognosis of COVID-19. T2DM with hyperglycemia are among factors that lead to elevated expression of angiotensin-converting enzyme 2 (ACE2) in lungs and other tissues; ACE2 is the cellular "receptor" and port of viral entry. The preexisting chronic inflammation with augmented inflammatory response to the infection and the increasing viral load leads to extreme systemic immune response ("cytokine storm") that is strongly associated with increased severity of COVID-19

(https://www.ncbi.nlm.nih.gov/pmc/articles/PMC7405270/). Using of $\alpha$-glucosidase inhibitors was found to be the potential protectant for severe events (https://www.ncbi.nlm.nih.gov/pmc/articles/PMC7543084/).HbAlc was an independent risk factor for poor outcomes in coronavirus disease 2019 patients

(https://www.ncbi.nlm.nih.gov/pmc/articles/PMC7675705/). Increased blood glucose level was correlated with the severity of lung involvement, as evidenced by certain chest CT parameters, and clinical prognosis in diabetic COVID19 patients. There was a positive correlation between blood glucose level (both HbAlc and FBG) on admission and lung lesions (https://www.ncbi.nlm.nih.gov/pmc/articles/PMC7792916/). Optimizing glycemic control strategies in COVID-19 patients during the early phase of hospitalization is related to good prognosis

(https://www.ncbi.nlm.nih.gov/pmc/articles/PMID:33479161/) (https://www.ncbi.nlm.nih.gov/pmc/articles/PMC7675481/) (https://www.ncbi.nlm.nih.gov/pmc/articles/PMID:33073361/)

In addition, there is no information on new hyperglycemia because of retrospective record-based study, and it is impossible to comment on its association with COVID-19 results.

2. The exact interaction between COVID-19 and DM that leads to the worsening of COVID-19 at the molecular level was not carried in this study. T2DM with hyperglycemia are among factors that lead to elevated expression of angiotensin- 
converting enzyme 2 (ACE2) in lungs and other tissues; ACE2 is the cellular "receptor" and port of viral entry (https://www.ncbi.nlm.nih.gov/pmc/articles/PMC7405270/). Dysregulation of the host immune response, especially reduced cellular immunity, plays an important role in the pathophysiology of COVID-19. Severe acute respiratory syndrome coronavirus 2 (SARS-CoV-2) can alter both the innate and adaptive immune responses (https://www.ncbi.nlm.nih.gov/pmc/articles/PMC7351676/). Reduced CD3+, CD4+, and CD8+ T lymphocyte counts may reflect the severity of COVID-19 (https://www.ncbi.nlm.nih.gov/pmc/articles/PMC7219428/). The authors only analyzed lymphocyte percentage on the severity and prognosis of COVID-19, and found that lymphocyte percentage has no significant impact on the severity and prognosis of COVID-19, which is inconsistent with reports in the literature that partially decreased lymphocyte subsets was closely related to disease progression and prognosis in COVID-19 patients. (https://www.ncbi.nlm.nih.gov/pmc/articles/PMC7802992/) (https://www.ncbi.nlm.nih.gov/pmc/articles/PMC7373679/) (https://www.ncbi.nlm.nih.gov/pmc/articles/PMC7711740/) (https://www.ncbi.nIm.nih.gov/pmc/articles/PMC7235581/) (https://www.ncbi.nlm.nih.gov/pmc/articles/PMC7521864/).

The authors did not analyze lymphocyte and subsets and its effect on the severity and prognosis of COVID-19, and also did not analyze the differences of lymphocyte and subsets between COVID-19 patients with and without diabetes or hypertension.

4. In this article no significant association between HTN and COVID-19 severity or mortality was found which is inconsistent with reports in the literature that hypertension could also greatly affect the prognosis of the COVID-19 (https://www.ncbi.nlm.nih.gov/pmc/articles/PMC7177098/)

(https://www.ncbi.nlm.nih.gov/pmc/articles/PMC7332452/)

, hypertension possess an apparent relation to COVID-19 severity

(https://www.ncbi.nlm.nih.gov/pmc/articles/PMC7340589/)

(https://www.ncbi.nlm.nih.gov/pmc/articles/PMC7737518/)

, and hypertension was associated with the severity and mortality of COVID-19

illness (https://www.ncbi.nlm.nih.gov/pmc/articles/PMC8149896/)

5. Since this was a retrospective record-based study, hypertension related information such as hypertension control level, duration of hypertension, classification and risk stratification of hypertension, and target organ damage were lost. In addition to considering the hypertension itself affecting the severity and prognosis of COVID-19, it is also necessary to consider above hypertension related factors affecting the severity and prognosis of COVID-19.

6. The sample size 813 of this study is not large enough to avoid bias in research results.

7. Several other parameters that have been previously shown to be associated with COVID-19 outcome such as obesity, inflammation markers were not considered. Diabetes mellitus and hypertension have chronic systemic low-grade inflammation. The preexisting chronic inflammation with augmented inflammatory response to the infection and the increasing viral load leads to extreme systemic immune response ("cytokine storm") that is strongly associated with increased severity of COVID-19 (https://www.ncbi.nlm.nih.gov/pmc/articles/PMC7405270/). 